Supplement of Atmos. Chem. Phys., 22, 2011-2027, 2022

https://doi.org/10.5194/acp-22-2011-2022-supplement

(C) Author(s) 2022. CC BY 4.0 License.

(c) (i)

Atmospheric
Chemistry
and Physics

Supplement of

\title{
Source-resolved variability of fine particulate matter and human exposure in an urban area
}

Pablo Garcia Rivera et al.

Correspondence to: Spyros N. Pandis (spyros@chemeng.upatras.gr)

The copyright of individual parts of the supplement might differ from the article licence. 
1 Table S1. Outer (CONUS) boundary condition concentrations of major aerosol species.

2

\begin{tabular}{lcccc}
\hline \multirow{2}{*}{ Component } & \multicolumn{4}{c}{ Concentration $\left(\mu \mathrm{g} \mathrm{m}^{-3}\right)$} \\
& West & East & South & North \\
\hline Nitrate & 0.01 & 0.01 & 0.03 & 0.03 \\
Ammonium & 0.14 & 0.25 & 0.24 & 0.16 \\
Sulfate & 0.64 & 1.12 & 0.81 & 0.68 \\
Elemental carbon & 0.04 & 0.05 & 0.09 & 0.03 \\
Organic aerosol (Winter) & 0.20 & 0.16 & 0.58 & 0.80 \\
Organic aerosol (Summer) & 0.80 & 0.80 & 0.80 & 0.80 \\
\hline
\end{tabular}

3

4 
5 Table S2. Comparison of total $\mathrm{PM}_{2.5}$ performance with the use of old surrogates and new

6 surrogates for onroad traffic and commercial cooking. Measurements from EPA-CSN

7 and low-cost sensors (RAMPs) withing the inner $1 \times 1 \mathrm{~km}$ modeling domain were used.

\begin{tabular}{ccccc}
\hline & \multicolumn{3}{c}{ February 2017 } & \\
\hline & \multicolumn{2}{c}{ Old Surrogates } & \multicolumn{2}{c}{ New Surrogates } \\
& EPA-CSN & RAMPs & EPA-CSN & RAMPs \\
\hline $\begin{array}{c}\text { Observed Average } \\
\left(\boldsymbol{\mu g} \mathbf{~ m}^{-3}\right)\end{array}$ & 10.38 & 11.65 & 10.38 & 11.65 \\
$\begin{array}{c}\text { Predicted Average } \\
\left(\boldsymbol{\mu} \mathbf{g ~ m}^{-3}\right)\end{array}$ & 10.36 & 11.32 & 10.52 & 13.50 \\
Error $\left(\boldsymbol{\mu g} \mathbf{~ m}^{-3}\right)$ & 2.87 & 4.12 & 3.02 & 5.12 \\
Fractional Error & 0.29 & 0.31 & 0.30 & 0.38 \\
Bias $\left(\boldsymbol{\mu g} \mathbf{~ m}^{-3}\right)$ & -0.02 & -0.33 & 0.18 & 1.85 \\
Fractional Bias & 0.06 & 0.08 & 0.07 & 0.24 \\
\hline
\end{tabular}

\section{July 2017}

\begin{tabular}{ccccc}
\hline & \multicolumn{2}{c}{ Old Surrogates } & \multicolumn{2}{c}{ New Surrogates } \\
& EPA-CSN & RAMPs & EPA-CSN & RAMPs \\
\hline $\begin{array}{c}\text { Observed Average } \\
\left(\boldsymbol{\mu} \mathbf{g ~ m}^{-3}\right)\end{array}$ & 11.24 & 12.58 & 11.24 & 12.58 \\
$\begin{array}{c}\text { Predicted Average } \\
\left(\boldsymbol{\mu} \mathbf{g ~ m}^{-3}\right)\end{array}$ & 7.13 & 7.98 & 7.23 & 8.83 \\
Error $\left(\boldsymbol{\mu g} \mathbf{~ m}^{-3}\right)$ & 4.70 & 5.32 & 4.67 & 4.89 \\
Fractional Error & 0.49 & 0.47 & 0.48 & 0.42 \\
Bias $\left(\boldsymbol{\mu g} \mathbf{~ m}^{-3}\right)$ & -4.11 & -4.61 & -4.01 & -3.76 \\
Fractional Bias & -0.41 & -0.37 & -0.39 & -0.27 \\
\hline
\end{tabular}

8 

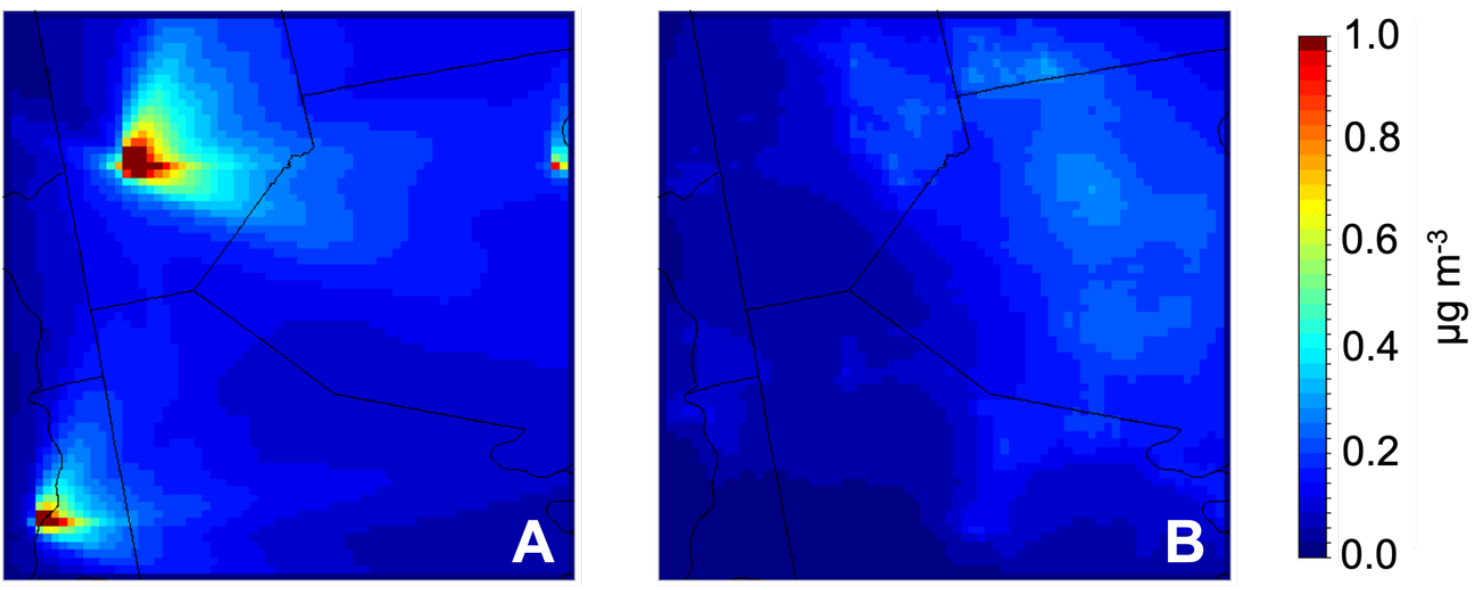

Figure S1 Average upper air concentration (13 simulated vertical layers above the ground 12 layer) of local PM $_{2.5}$ from (A) power generation and (B) biomass burning in February 2017. 13 

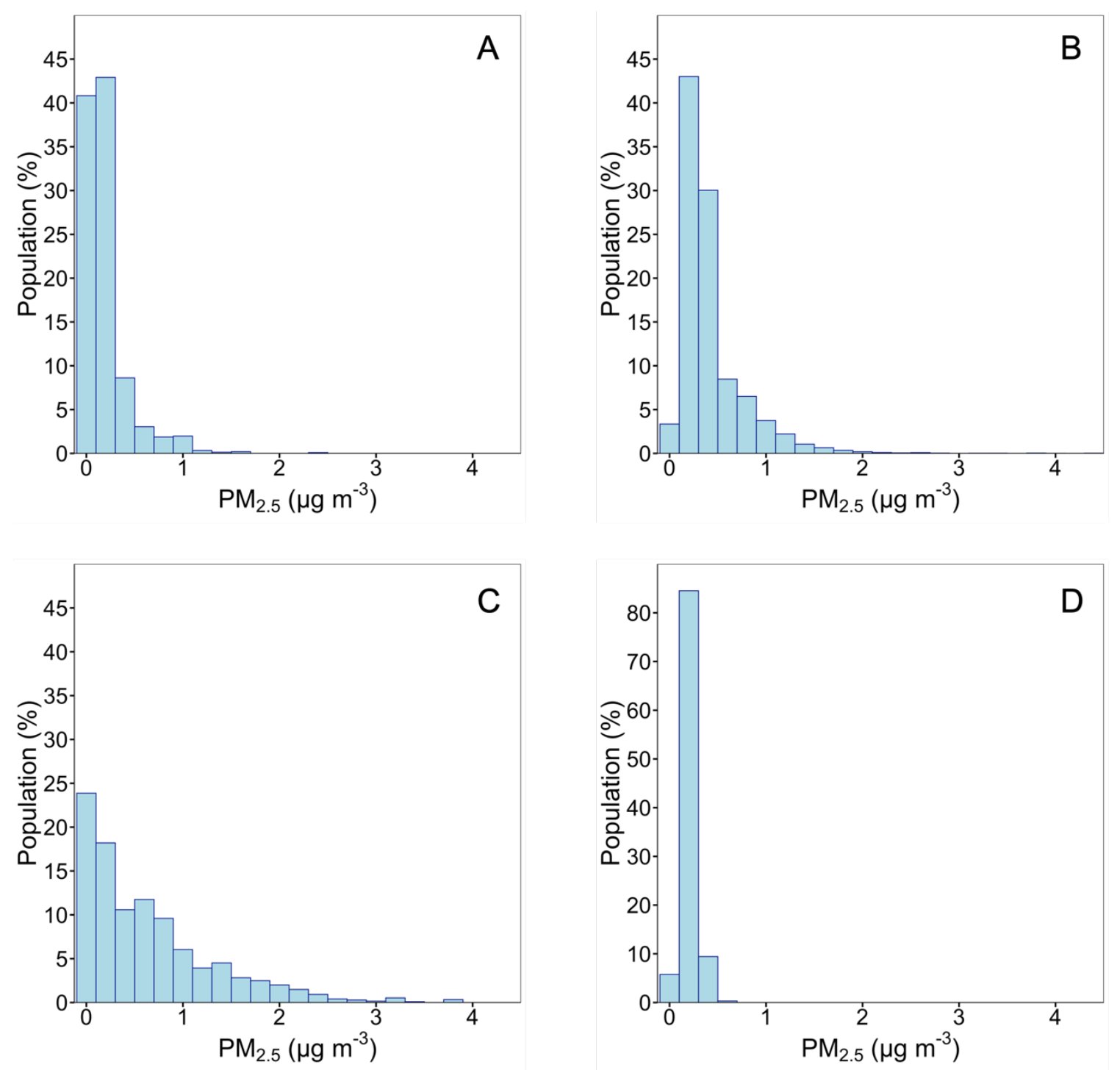

Figure S2 Population exposure histograms of the contribution to $\mathrm{PM}_{2.5}$ concentrations 17 from (A) commercial cooking, (B) industrial, (C) on-road traffic and (D) power generation 18 sources during February 2017. A different scale for population is used for the distribution 19 from power generation. 

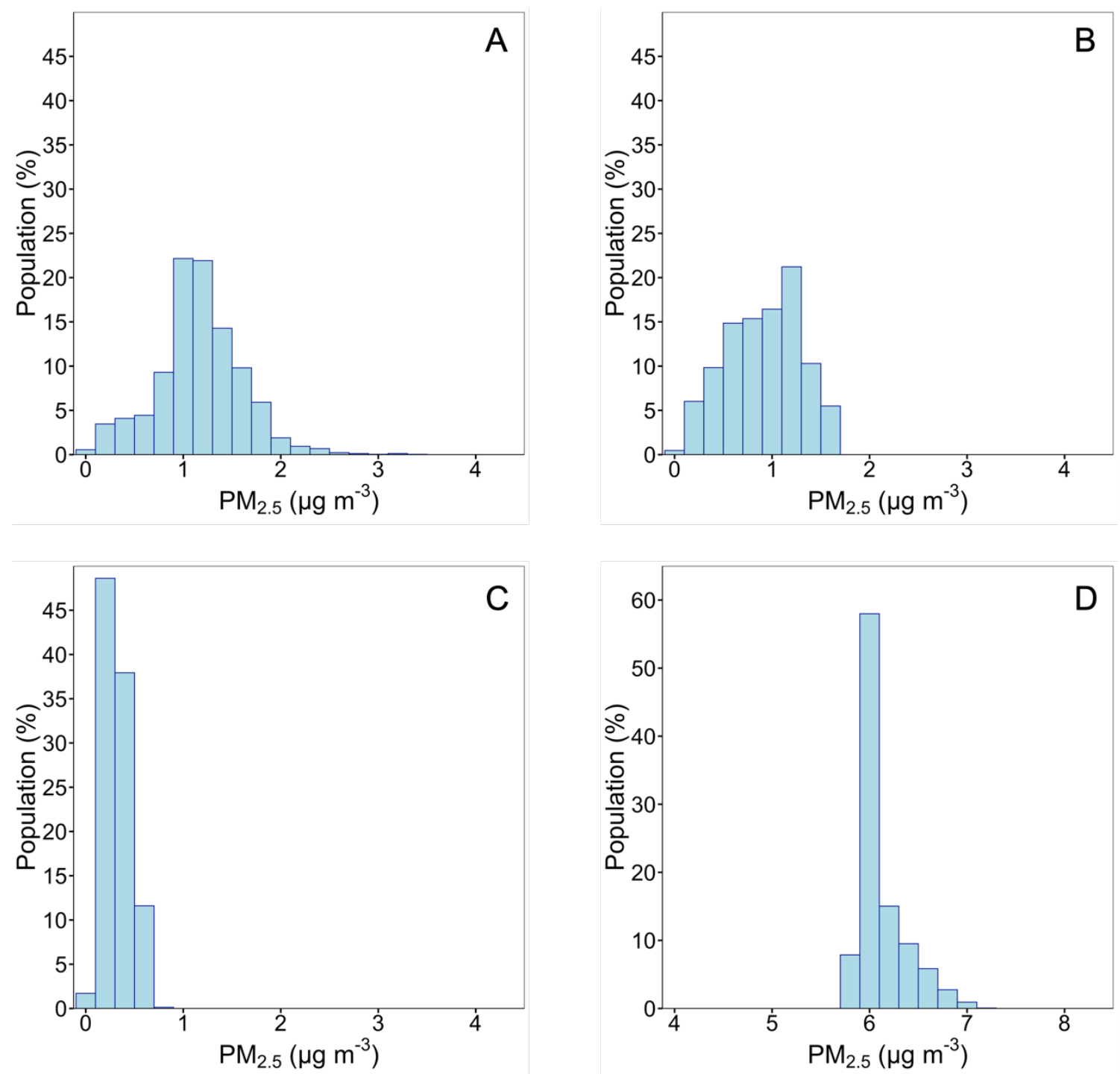

24 Figure S3 Population exposure histograms of the contribution to $\mathrm{PM}_{2.5}$ concentrations 25 from (A) biomass burning, (B) miscellaneous area sources and (C) all other sources during 26 February 2017. Contributions from long-range transport (D) are shown with a different 27 concentration scale. 

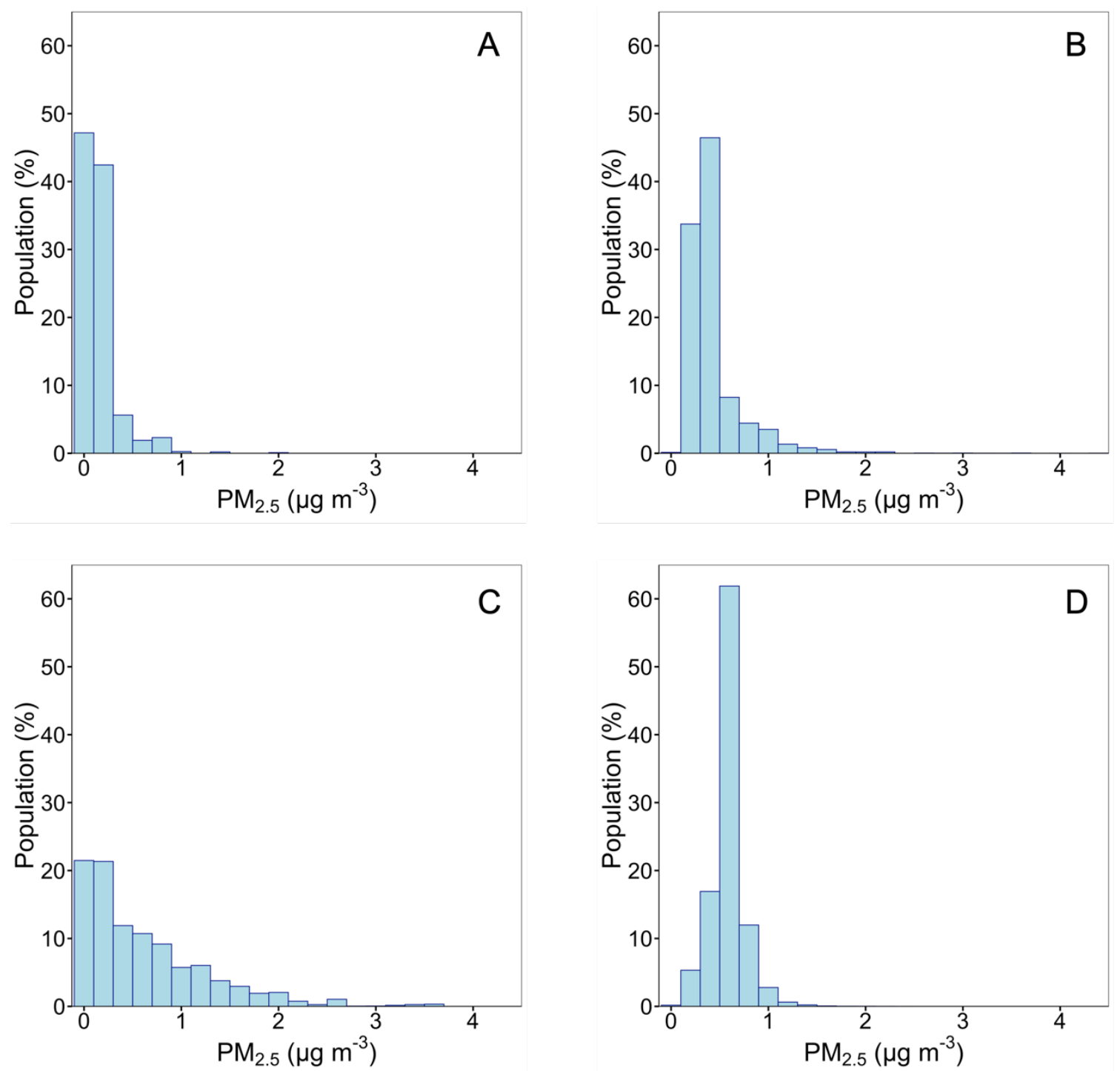

Figure S4 Population exposure histograms of the contribution to $\mathrm{PM}_{2.5}$ concentrations from (A) commercial cooking, (B) industrial, (C) on-road traffic and (D) power generation sources during July 2017. 

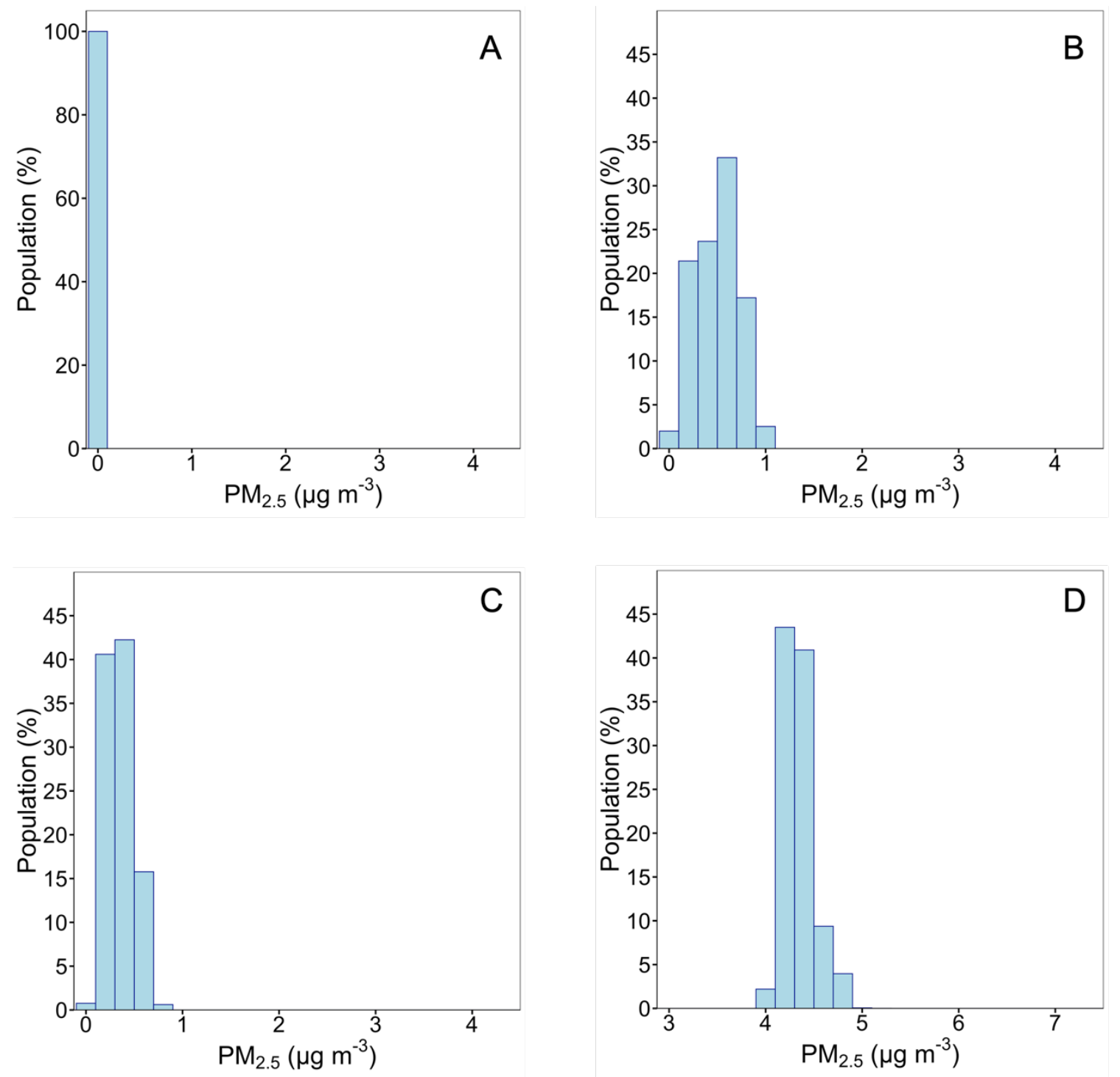

Figure S5 Population exposure histograms of the contribution to $\mathrm{PM}_{2.5}$ concentrations

39 from (A) biomass burning, (B) miscellaneous area sources and (C) all other sources during

40 July 2017. Contributions from long-range transport (D) are shown with a different 41 concentration scale. 


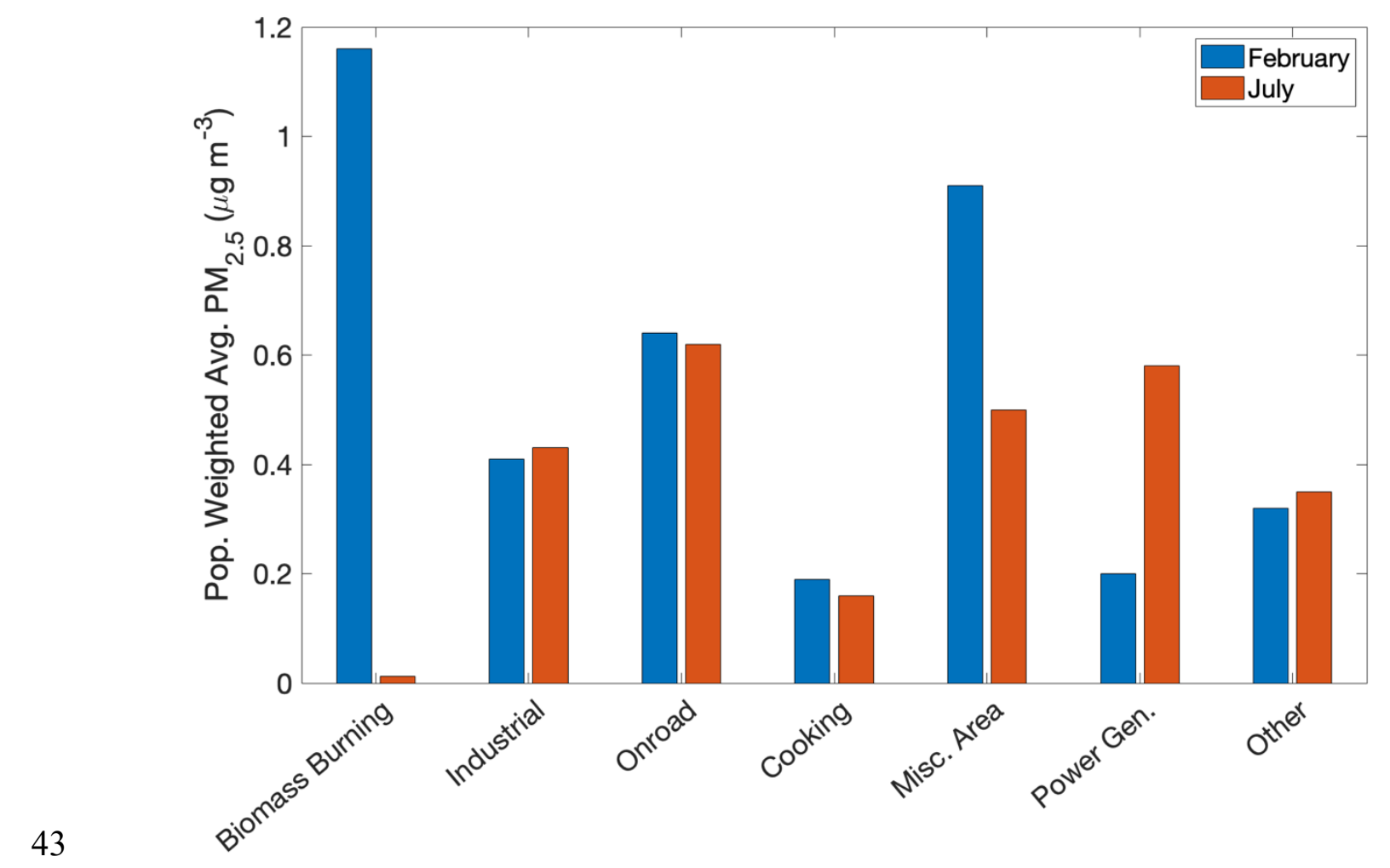

44 Figure S6 Absolute contributions from local sources to population weighted total $\mathrm{PM}_{2.5}$ 45 concentration for February and July 2017

46

47 